\title{
Structural equation modelling the relationship between anti-fungal prophylaxis and Pseudomonas bacteremia in ICU patients.
}

James Hurley ( $\sim$ hurleyjc@unimelb.edu.au )

University of Melbourne

\section{Research Articles}

Keywords: topical antibiotics, candidemia, generalized structural equation modelling, anti-fungal, Pseudomonas bacteremia

Posted Date: September 30th, 2021

DOI: https://doi.org/10.21203/rs.3.rs-929933/v1

License: (c) (1) This work is licensed under a Creative Commons Attribution 4.0 International License.

Read Full License 


\section{Abstract \\ Purpose}

Animal models implicate candida colonization facilitating invasive bacterial infections. The clinical relevance of this microbial interaction remains undefined. Observations from studies of anti-septic, antibiotic, anti-fungal, and non-decontamination-based interventions to prevent ICU acquired infection collectively serve as a natural experiment.

\section{Methods}

Three candidate generalized structural equation models (GSEM), with Candida and Pseudomonas colonization as latent variables, were confronted with blood culture and respiratory tract isolate data derived from 460 groups from 279 studies including studies of combined antibiotic and antifungal exposures within selective digestive decontamination (SDD) interventions.

\section{Results}

Introducing an interaction term between Candida colonization and Pseudomonas colonization substantially improved GSEM model fit. Model derived coefficients for singular exposure to anti-septic agents $(-1.23 ;-2.1$ to -0.32$)$, amphotericin $(-1.78 ;-2.79$ to -0.78$)$ and topical antibiotic prophylaxis (TAP; $+1.02 ;+0.11$ to +1.93 ) versus Candida colonization were similar in magnitude but contrary in direction. By contrast, the model-derived coefficients for singular exposure to TAP, as with anti-septic agents, versus Pseudomonas colonization were weaker or non-significant. Singular exposure to amphotericin would be predicted to more than halve candidemia and Pseudomonas bacteremia incidences versus literature benchmarks for absolute differences of approximately one percentage point or less.

\section{Conclusion}

GSEM modelling of published data supports the postulated interaction between Candida and Pseudomonas colonization towards promoting bacteremia among ICU patients. The model implicates that anti-fungal agents have greater impact in preventing bacteremia versus TAP, which has no impact.

\section{Introduction}

Animal models implicate candida colonization facilitating invasive bacterial infections $[1,2]$. Which of over 600 catalogued immunological, biochemical, metabolic and mechanical processes might underlie this interaction between Pseudomonas aeruginosa and Candida albicans and, moreover, whether it has clinical relevance, remain challenging to investigate [3-5]. 
Conceptually, clinical investigation of this postulated interaction would manipulate candida colonization among patients and measure the resulting bacteremia incidence [6-8]. Such a manipulation would be logistically complex. Additional challenges to such an approach are that the blood stream infection (BSI) endpoints are generally uncommon or rare, the key body site location of any postulated interaction, whether the oropharynx or elsewhere, remains unclear and measuring colonization, whether bacterial or candida, is problematic. Moreover, whether changes in the metabolic activity, hyphal growth or the mere viable count of Candida colonisation drives any interaction remains moot.

A novel approach uses the collective observations from numerous studies of assorted and variously formulated anti-septic, antibiotic, anti-fungal, and non-decontamination-based interventions in the prevention of ICU acquired infections such as ventilator associated pneumonia (VAP) [9-17]. Candidemia and bacteremia incidences, being occasional secondary endpoints within these studies, in association with varying Candida colonisation occurring as a bystander process to the study intervention, serve as a natural experiment.

Selective digestive decontamination (SDD), a widely studied antibiotic-based regimen, combines antibiotic and antifungal exposures. Moreover, the SDD concept invokes population effects and several SDD studies in the ICU setting avoid these anticipated contextual effects by using either non-concurrent or no control group patients [18-22]. These contextual effects need to be considered in any analysis.

The objectives here are threefold. Firstly, to recapitulate the study level evidence for various ICU infection prevention interventions versus each of VAP and BSI with Candida or Pseudomonas. Second, to develop models of colonization and infection based on the postulated interaction between Candida and Pseudomonas colonization as impacted by various infection prevention interventions and confront the models using group level infection data using GSEM modelling. Thirdly, to estimate the relative impacts of anti-septic, antibiotic, and specific anti-fungal agents as singular or compound exposures on bacteremia and candidemia within the optimal GSEM model.

\section{Materials And Methods}

Being an analysis of published work, ethics committee review of this study was not required.

\section{Study selection and decant of groups}

The literature search and study decant used here is as described previously [23-25] and is detailed in Fig. 1. The key inclusion criterion, being patient groups requiring prolonged ( $>24$ hours) ICU stay within studies of ICU infection prevention interventions, was expanded to include studies with group level Candida, and Pseudomonas infection data. The studies were streamed into type of infection prevention intervention, being non-decontamination based, anti-septic based, antibiotic based or single anti-fungal (SAF) based methods. Studies without ICU infection prevention interventions were sourced to provide summary benchmark incidence data. Most of the studies had been cited in systematic reviews with 
additional studies being found by snowball sampling using the 'Related articles' function within Google Scholar [26].

The various study level and group level data were extracted for tabulation and to serve as indicator variables in the GSEM models.

\section{Visual benchmarking}

Scatter plots of VAP and blood stream infections in association with Candida and Pseudomonas infection were generated to facilitate a visual survey of the entire data versus benchmarks as derived from the literature.

\section{Estimation of summary effect sizes}

As study events were rare, summary effect sizes were derived using the Peto's log odds ratio [27]. The Stata meta command was used for deriving summary effect sizes, the associated measures of betweenstudy heterogeneity and the caterpillar plots which display the results of individual studies.

\section{GSEM models: Measurement components}

The incidences of VAP and blood stream infections in association with Candida Pseudomonas were extracted. As Candida is generally not considered a cause of VAP, the count of Candida as a respiratory tract (RT Candida) isolate among patients with suspected VAP was recorded. These counts were each transformed to proportions using the number of patients with prolonged ( $>24$ hours) ICU stay as the denominator. In the GSEM models, Candida and Pseudomonas colonization are each latent variables.

\section{GSEM models: indicator variables}

The following data constitute the indicator variables of the GSEM models; origin from trauma ICU's, being defined here as an ICU with $>50 \%$ of admissions being for trauma, whether more than $90 \%$ of patients of the group received more than 24 hours of MV, and a mean (or median) length of ICU stay (ICU-LOS) for the group greater than 7 days. In the extraction of MV percentages, if this was not stated for any group, the percentage receiving MV of less than $90 \%$ was assumed. In the extraction of ICU-LOS data from the studies, surrogate measures including mean (or median) length of mechanical ventilation were taken if the length of ICU-LOS was not available in order to generate a binary variable of ICU-LOS of greater or less than 7 days.

Also, the presence of any of the following group wide risk factors for candidemia (CRF) and invasive Candida infection were noted; liver transplantation or liver failure, use of parenteral nutrition, surgery for intestinal perforation, pancreatitis, and being colonized with Candida, however that was defined. Antiseptic exposure included use of agents such as chlorhexidine, povidone-iodine and iseganan regardless of whether the application was to the oropharynx, by tooth-brushing or by body-wash.

Antibiotic-based interventions were classified as follows. Topical antibiotic prophylaxis (TAP) is defined without regard to the specific antibiotic constituents and whether application was to the oropharynx and 
or gastrointestinal tract. Protocolized parenteral antibiotic prophylaxis (PPAP) is use of prophylactic parenteral antibiotic as dictated by the study protocol whether to intervention group alone or to both control and intervention groups (duplex studies). Exposure to anti-fungal prophylaxis was identified whether as a single agent or in combination with antibiotic-based interventions as within SDD or selective oropharyngeal decontamination (SOD) regimens.

\section{Structural equation modelling}

In the GSEM models, the VAP and blood stream infection proportion data, serve as the measurement components, the group level exposure parameters serve as the indicator variables and colonization with each of Candida and Pseudomonas, being represented as latent variables, link the indicator and measurement components.

Three candidate GSEM models were developed. The first two, with and without the inclusion of an interaction terms between the latent variables, being Candida colonization and Pseudomonas colonization and the third with the addition of concurrent control group membership within an antibioticbased study as an indicator variable.

Because the observations are clustered by study, study identifiers were used in the models to enable generation of robust variance covariance matrices of the coefficient estimate parameters. The GSEM model with the lowest Akaike's information criterion (AIC) score was selected as having parsimony and optimal fit from among the candidate models using the 'GSEM' command in Stata (Stata 17, College Station Texas, USA) [28]. The post model predictions were obtained using the command ' $n$ lcom' to obtain nonlinear combinations of estimators

\section{Results}

\section{Characteristics of the studies}

Of the 279 studies identified by the search, most were sourced from 23 systematic reviews (Table 1; Fig 1), most were published between 1990 and 2010 and most had a mean ICU-LOS exceeding seven days. Twelve studies had more than one type of intervention group and 15 studies had either more than one or no control group. The majority of groups from studies of infection prevention interventions had less than 150 patients per group versus more than 150 patients in the observational studies.

The incidence of BSI (Fig 2) and VAP (Fig S1 - S2) with each of Candida and Pseudomonas ranged approximately 100 -fold across the various control and intervention groups of the 279 studies. In each case, the incidence was approximately $50 \%$ higher among concurrent control groups within studies where intervention groups received TAP versus a benchmark derived from observational groups. The candidemia incidence was higher among groups from SAF studies as patient selection for most of these studies was commonly based on presence of CRF. 
Bacteremia and candidemia incidences were generally lower among studies of anti-septic interventions as there was a lower proportion with respect to each of the following versus other study categories: mean length of stay less than 7 days, trauma ICU's, patient selection for CRF and use of MV for $>48$ hours.

\section{Summary effect sizes}

Among the summary prevention effects, the strongest was for antibiotic-based interventions versus Pseudomonas VAP (odds ratio $0.33 ; 95 \% \mathrm{Cl} ; 0.26$ to 0.42 ) (Table 1 ). In the prevention of candidemia, the indicative summary effect size for the SAF and antibiotic-based interventions were similar. All other summary effect estimates for all other interventions versus the VAP, bacteremia, or candidemia endpoints were either weaker or not significant (Table 1; Fig S3 - S6). Of note, surprisingly, no significant prevention effect for Pseudomonas bacteremia was apparent for any intervention (Table 1; Fig S3 - S6).

\section{GSEM modelling}

Three GSEM models of the relationship between various group level exposures on Candida and Pseudomonas colonization as latent variables were evaluated for fit and parsimony (Table S6; Fig S7 S8; Fig 3). The introduction of firstly Candida colonization as a cofactor towards Pseudomonas colonization, and then, membership of concurrent control groups within studies of antibiotic-based interventions as an indicator variable, each improved the model fit towards the optimal model (Fig 3).

In the optimal model, the coefficients for singular exposure to anti-septic agents $(-1.23 ;-2.1$ to -0.32$)$, amphotericin $(-1.78 ;-2.79$ to -0.78$)$ and topical antibiotic prophylaxis (TAP; $+1.02 ;+0.11$ to +1.93 ) versus Candida colonization were similar in magnitude but contrary in direction.

In all models, group wide exposure to CRF, anti-septics and singular exposures to each of TAP and antifungals, although less so for nystatin, displayed strong and significant associations with the Candida colonization latent variable, and these were generally consistent across the three models. By contrast, the same exposures versus Pseudomonas colonization were generally weaker, less consistent between models and variably significant.

\section{Post GSEM modelling predictions}

Post model predictions of Pseudomonas bacteremia and Candidemia incidences were estimated for a putative group of non-trauma ICU patients with group mean LOS greater than seven days and without patient selection for CRF. Predictions were made for various combination and singleton group wide exposures to anti-septic agents, TAP, PPAP, nystatin and amphotericin versus a benchmark derived for an equivalent putative non-concurrent control group (Fig 4). In every case, singleton exposure to either amphotericin or to anti-septics outperformed singleton exposure to TAP towards lower predicted bacteremia incidences. Exposure to TAP combined with amphotericin, but not nystatin, was associated with significantly lower predicted bacteremia incidences versus benchmark. 


\section{Discussion}

There were three objectives here. Firstly, the study level evidence for various ICU infection prevention interventions toward preventing VAP and BSI derived here broadly recapitulates prior summary estimates among systematic reviews in the literature (Table S6) [9-17]. The effect sizes are indicative as each category includes broadly selected studies, with some having compound interventions, such as TAP combined with an anti-fungal, together with without PPAP given to either or both of control and intervention groups in antibiotic-based studies. The selection of studies in the summaries here is congruous with that in the literature summaries. Of note, the concurrent control groups within antibioticbased intervention studies have unusually high incidences for several end-points that are unexplained in previous meta-regression models, taking into account several other group level variables not included here, such as year of publication, and likely represents a contextual effect [29-30].

The second objective is the development of GSEM models. The optimal model includes both the postulated interaction between Candida and Pseudomonas colonization and the contextual influences of TAP on concurrent control groups. In confronting the optimal GSEM model with published group level infection data, the anti-fungal agents, such as azoles and amphotericin, and anti-septic agents, each showed strong prevention effects versus Candida colonization. By contrast, TAP as a singular exposure versus Pseudomonas colonization and versus Candida colonization demonstrated effects that were weaker and variable in direction and significance.

Thirdly, in post GSEM model predictions, the estimated effects of singular exposure to topical amphotericin and anti-septic agents would each more than halve the incidence of candidemia and Pseudomonas bacteremia, although for absolute differences being approximately one percentage point or less. These differences would be challenging to detect. For example, a cluster-randomized trial demonstrating halving in Pseudomonas bacteremia incidence from $1 \%$ in the control group to $0.5 \%$ in the intervention group would need to enrol over 2,000 ICU's each providing 500 patients per arm to provide $80 \%$ power.

By contrast, singular TAP exposure, with or without PPAP in combination, was either neutral or promoted bacteremia and candidemia incidence. Of note, the most common PPAP among SDD studies is cefotaxime, which is not generally active against Pseudomonas. That antibiotic exposure in the ICU environment paradoxically promotes Pseudomonas acquisition is a finding with precedent [31, 32]. Moreover, bacterial colonization rebounds following cessation of TAP with effects on the whole-of-ICU population [33]. Whether rebound contributes to colonization pressure, and the contextual effects of TAP exposure, needs to be further examined [34].

The observations here are paradoxical. On the one hand, antibiotic-based interventions show strong prevention effects against VAP that is most apparent for Pseudomonas VAP together with a halving in candidemia among studies of antibiotic-based interventions. 
On the other hand, despite these two strong prevention effects of antibiotic-based interventions, there is insignificant prevention of Pseudomonas bacteremia. Moreover, the incidences of candidemia and bacteremia are generally higher among the concurrent control groups of antibiotic-based studies.

These observations, while paradoxical, potentially explain how ICU-acquired gram-negative bacteremia might occur seemingly without preceding colonization $[35,36]$. They also could account for the bacteremia prevention effects observed in large studies of antibiotic-based interventions of SDD regimens containing topical polymyxin and tobramycin combined with amphotericin as the anti-fungal $[20,21]$, whereas the largest SDD study, where the same TAP regimen was combined with nystatin [22], failed to demonstrate any prevention effects against any bacteremia, or candidemia, end-points.

The SEM technique is an emerging method among critical care and infection pathogenesis research that enables group level modelling of multiple simultaneously observed variables [37, 38]. This technique enables the testing of concepts that infer relationships between observed variables mediated through latent variables. GSEM allows generalized linear response functions in addition to the linear response functions allowed by non-generalized SEM. A strength of GSEM modelling is the ability to incorporate observations from clusters with missing observations under the assumption of missing at random. This enables the inclusion of groups from studies either lacking control groups or providing data for only some end-points.

\section{Limitations}

There are multiple limitations and cautions in the interpretation of the modelling here. The GSEM is a group level modelling of two latent variables, Candida and Pseudomonas within a postulated model of interaction. These latent variables and the coefficients derived in the GSEM are indicative only. They have no counterpart at the level of any one patient or study and cannot be directly measured.

The second limitation is that there was no ability nor purpose to adjust for the underlying patient level risk. There was considerable heterogeneity in the interventions, populations, and study designs among the studies meeting intentional broad inclusion criteria. This stands in contrast to the technique of network meta-analysis (NMA) applied to data obtained from studies meeting tight inclusion criteria in order to satisfy transitivity assumptions. With transitivity, a NMA enables comparisons of multiple interventions as allocated to comparable patient groups within randomized controlled trials towards estimating patient level effects from study level data. By contrast, the GSEM technique enables modelling of group-level associations towards deriving group level inferences among patient groups experiencing compound exposures within studies assembled with less stringent inclusion criteria. This allows the inclusion of concurrent control groups from antibiotic-based studies where contextual effects invalidate the transitivity assumption.

The third limitation is that the GSEM model is deliberately simplistic. There are only limited numbers of key group level factors, the exposures are entered as binary variables, and with interaction between the 
latent variables being the only interactions tested. In reality, the relationships between expoures and outcomes will likely be graded and complex with potentially compound expoure interactions.

Finally, the various regimens of antibiotic-based, anti-septic and anti-fungal interventions used within the various studies have been considered as similar within each category. This is a deliberate simplification as some, for example some SAF interventions were administered parenterally rather than topically. Also, the intensity and duration of application, and the body site targeted by the various interventions, varied among the studies and have not been modelled. On the other hand, a strength of this analysis is that the various compound interventions, as for example within SDD regimens comprising TAP, PPAP, and antifungal components, are factorized towards estimating their separate singleton associations on the latent variables within the GSEM model.

\section{Conclusion}

GSEM modelling of Pseudomonas and candida colonization, each as latent variables versus group level exposures, demonstrates complex and paradoxical relationships that would not be apparent in any single study examined in isolation nor within a summary effect of the collective studies as derived by conventional meta-analytic modelling. The model provides support to the postulated interaction between candida and Pseudomonas colonization in facilitating invasive Pseudomonas infections. Anti-fungal interventions have potentially stronger prevention impact on Pseudomonas bacteremia, mediated via candida colonization, than does singleton TAP exposures, which has no impact or PPAP, which promotes Pseudomonas bacteremia.

\section{Abbreviations}

BSI, blood stream infection; CRF, Candida risk factors; ICU, Intensive Care Unit; MV, Mechanical Ventilation ; SAF, single anti-fungal; VAP ventilator associated pneumonia; PPAP, Protocolized parenteral antibiotic prophylaxis; RT Candida; Respiratory tract Candida; SDD, Selective Digestive Decontamination; TAP, topical antibiotic prophylaxis; GSEM, generalized structural equation models.

\section{Declarations}

\section{Competing interests}

The author declares that he has no competing interests.

\section{Author contributions}

As sole author, $\mathrm{JH}$ produced the design of the study, performed the statistical analysis and wrote the manuscript. JH read and approved the final manuscript.

\section{Funding}


This work was supported by the Australian Government Department of Health and Ageing through the Rural Clinical Training and Support (RCTS) program.

\section{Availability of data and materials}

All data generated or analysed during this study are included in this published article and the electronic supplementary material (ESM).

\section{References}

1. Bergeron AC, Seman BG, Hammond JH, Archambault LS, Hogan DA, Wheeler RT (2017) Candida albicans and Pseudomonas aeruginosa interact to enhance virulence of mucosal infection in transparent zebrafish. Infection immun 85(11):e00475-e00417

2. Grainha T, Jorge P, Alves D, Lopes SP, Pereira MO (2020) Unravelling Pseudomonas aeruginosa and Candida albicans communication in coinfection scenarios: insights through network analysis. Front cell infect microbial 10

3. Albert M, Williamson D, Muscedere J, Lauzier F, Rotstein C, Kanji S et al (2014) Candida in the respiratory tract secretions of critically ill patients and the impact of antifungal treatment: a randomized placebo controlled pilot trial (CANTREAT study). Intensive Care Med 40:1313-1322

4. Lindau S, Nadermann M, Ackermann H, Bingold TM, Stephan C, Kempf VA et al (2015) Antifungal therapy in patients with pulmonary Candida spp. colonization may have no beneficial effects. $J$ Intensive Care 3(1):31

5. Timsit JF, Schwebel C, Styfalova L, Cornet M, Poirier P, Forrestier C et al (2019) Impact of bronchial colonization with Candida spp. on the risk of bacterial ventilator-associated pneumonia in the ICU: the FUNGIBACT prospective cohort study. Intensive Care Med 45(6):834-843

6. Azoulay E, Timsit JF, Tafflet M, de Lassence A, Darmon M, Zahar JR et al (2006) Candida colonization of the respiratory tract and subsequent pseudomonas ventilator-associated pneumonia. Chest 129(1):110-117

7. Nseir S, Jozefowicz E, Cavestri B, Sendid B, Di Pompeo C, Dewavrin F et al (2007) Impact of antifungal treatment on Candida-Pseudomonas interaction: a preliminary retrospective casecontrol study. Intensive Care Med 33(1):137-142

8. Huang D, Qi M, Hu Y, Yu M, Liang Z (2019) The impact of Candida spp airway colonization on clinical outcomes in patients with ventilator-associated pneumonia: A systematic review and meta-analysis. Am J Infection Control pii: S0196-6553(19)30956-3. doi: 10.1016/j.ajic.2019.11.002

9. Minozzi S, Pifferi S, Brazzi L, Pecoraro V, Montrucchio G, D'Amico R (2021) Topical antibiotic prophylaxis to reduce respiratory tract infections and mortality in adults receiving mechanical ventilation. Cochrane Database Syst Rev Issue 1 Art No: CD000022.

DOI:10.1002/14651858.CD000022.pub4 
10. Pileggi C, Bianco A, Flotta D, Nobile CG, Pavia M (2011) Prevention of ventilator-associated pneumonia, mortality and all intensive care unit acquired infections by topically applied antimicrobial or antiseptic agents: a meta-analysis of randomized controlled trials in intensive care units. Crit Care 15:R155

11. Silvestri L, Van Saene HK, Casarin A, Berlot G, Gullo A (2008) Impact of selective decontamination of the digestive tract on carriage and infection due to Gram-negative and Gram-positive bacteria: a systematic review of randomised controlled trials. Anaesthesia Intens Care 36(3):324-338

12. Hurley JC (1995) Prophylaxis with enteral antibiotics in ventilated patients: Selective decontamination or selective cross-infection? Antimicrob Agents Chemother 39:941-947

13. Silvestri L, Van Saene HK, Milanese M, Gregori D, Gullo A (2007) Selective decontamination of the digestive tract reduces bacterial bloodstream infection and mortality in critically ill patients. Systematic review of randomized, controlled trials. J Hosp Infect 65(3):187-203

14. Silvestri L, Weir WI, Gregori D, Taylor N, Zandstra DF, van Saene JJ, van Saene HK (2017) Impact of Oral Chlorhexidine on Bloodstream Infection in Critically III Patients: Systematic Review and MetaAnalysis of Randomized Controlled Trials. J Cardiothoracic Vasc Anesthesia 31(6):2236-2244

15. Labeau SO, Van de Vyver K, Brusselaers N, Vogelaers D, Blot SI (2011) Prevention of ventilatorassociated pneumonia with oral antiseptics: a systematic review and meta-analysis. Lancet Infect Dis 11:845-854

16. Klompas M, Speck K, Howell MD, Greene LR, Berenholtz SM (2014) Reappraisal of routine oral care with chlorhexidine gluconate for patients receiving mechanical ventilation: systematic review and meta-analysis. JAMA Intern Med 174(5):751-761

17. Silvestri L, Van Saene HK, Milanese M, Gregori D (2005) Impact of selective decontamination of the digestive tract on fungal carriage and infection: systematic review of randomized controlled trials. Intensive Care Med 31:898-910

18. Hurley JC (2021) Selective digestive decontamination, a seemingly effective regimen with individual benefit or a flawed concept with population harm? Crit Care 25(1):1-0

19. Stoutenbeek CP, Van Saene HK, Miranda DR et al (1984) The effect of selective decontamination of the digestive tract on colonisation and infection rate in multiple trauma patients. Intensive Care Med 10:185-192

20. de Smet AM, Hopmans TE, Minderhoud AL, Blok HE, Gossink-Franssen A, Bernards AT, Bonten MJ (2009) Decontamination of the digestive tract and oropharynx: hospital acquired infections after discharge from the intensive care unit. Intens Care Med 35(9):1609

21. Oostdijk EAN, Kesecioglu J, Schultz MJ et al (2017)Notice of Retraction and Replacement: Oostdijk et al (2014) Effects of Decontamination of the Oropharynx and Intestinal Tract on Antibiotic Resistance in ICUs: A Randomized Clinical Trial. JAMA. 312(14):1429-1437. JAMA 2017

22. Wittekamp BH, Plantinga NL, Cooper BS et al (2018) Decontamination strategies and bloodstream infections with antibiotic-resistant microorganisms in ventilated patients: a randomized clinical trial. JAMA 320:2087-2098 
23. Hurley JC (2015) ICU-acquired candidemia within selective digestive decontamination studies: a meta-analysis. Intensive Care Med 41(11):1877-1885

24. Hurley JC (2020) Structural equation modelling the 'control of gut overgrowth' in the prevention of ICU acquired Gram-negative infection. Crit Care 24:189

25. Hurley JC (2016) Impact of selective digestive decontamination on respiratory tract Candida among patients with suspected ventilator-associated pneumonia. A meta-analysis. Eur J Clin Microbiol Infect Dis 35(7):1121-1135

26. Goodman LA (1961) Snowball sampling. Ann Math Statistics, $148-70$

27. Peto R, Pike MC, Armitage P, Breslow NE, Cox DR, Howard SV, Mantel N, McPherson K, Peto J, Smith PG (1977) Design and analysis of randomized clinical trials requiring prolonged observation of each patient. II. Analysis and examples. Br J Cancer 35:1-39. https://doi.org/10.1038/bjc.1977.1

28. Stata corporation (2021) Stata structural equation modelling reference manual, in Stata 17 documentation. College Station, TX, USA. https://www.stata.com/bookstore/structural-equationmodeling-reference-manual/. Accessed 06 July 2021

29. Hurley JC (2018) Incidences of Pseudomonas aeruginosa-associated ventilator-associated pneumonia within studies of respiratory tract applications of polymyxin: testing the Stoutenbeek concurrency postulates. Antimicrob Agents Chemother 62(8):e00291-e00218

30. Hurley JC (2018) Unusually high incidences of Pseudomonas bacteremias within topical polymyxin based decolonization studies of mechanically ventilated patients: benchmarking the literature. Open Forum Infect Dis 5(11):ofy256

31. Venier AG, Leroyer C, Slekovec C, Talon D, Bertrand X, Parer S, Alfandari S, Guerin JM, Megarbane B, Lawrence C, Clair B (2014) Risk factors for Pseudomonas aeruginosa acquisition in intensive care units: a prospective multicentre study. J Hosp Infect 88(2):103-108

32. Hoang S, Georget A, Asselineau J, Venier AG, Leroyer C, Rogues AM, Thiébaut R (2018) Risk factors for colonization and infection by Pseudomonas aeruginosa in patients hospitalized in intensive care units in France. PloS one 13(3):e0193300

33. Tetteroo, Geert WM, Johan HT, Wagenvoort, Hajo A, Bruining (1994) Bacteriology of selective decontamination: efficacy and rebound colonization. J Antimicrob Chemother 34:139-148

34. Boyer A, Doussau A, Thiébault R, Venier AG, Tran V, Boulestreau H, Bébéar C, Vargas F, Hilbert G, Gruson D, Rogues AM (2011) Pseudomonas aeruginosa acquisition on an intensive care unit: relationship between antibiotic selective pressure and patients' environment. Crit Care 15(1):1-0

35. Frencken JF, Wittekamp BH, Plantinga NL, Spitoni C, van de Groep K, Cremer OL, Bonten MJ (2017) Associations Between Enteral Colonization With Gram-Negative Bacteria and Intensive Care UnitAcquired Infections and Colonization of the Respiratory Tract. Clin Infect Dis 66(4):497-503

36. Oostdijk EA, de Smet AM, Kesecioglu J, Bonten MJ (2011) The role of intestinal colonization with gram-negative bacteria as a source for intensive care unit-acquired bacteremia. Crit Care Med 39(5):961-966 
37. Carver S, Beatty JA, Troyer RM, Harris RL, Stutzman-Rodriguez K, Barrs VR et al (2015) Closing the gap on causal processes of infection risk from cross-sectional data: structural equation models to understand infection and co-infection. Parasites Vectors 8(1):658

38. Bojan M, Duarte MC, Ermak N, Lopez-Lopez V, Mogenet A, Froissart M (2016) Structural equation modelling exploration of the key pathophysiological processes involved in cardiac surgery-related acute kidney injury in infants. Crit Care 20(1):1-0

\section{Tables}

\section{Table 1. Characteristics of studies}




\begin{tabular}{|c|c|c|c|c|c|}
\hline & Observational & $\begin{array}{l}\text { Non- } \\
\text { decontamination }\end{array}$ & $\begin{array}{l}\text { Topical } \\
\text { anti- } \\
\text { septic }\end{array}$ & $\begin{array}{l}\text { Antibiotic } \\
\text { based }\end{array}$ & $\begin{array}{l}\text { Single } \\
\text { anti- } \\
\text { fungal }\end{array}$ \\
\hline \multicolumn{6}{|l|}{ Study characteristics } \\
\hline Listing & Table S1 & Table S2 & $\begin{array}{l}\text { Table } \\
\text { S3 }\end{array}$ & Table S4 & $\begin{array}{l}\text { Table } \\
\text { S5 }\end{array}$ \\
\hline Number of studies ${ }^{d}$ & 142 & 44 & 18 & 61 & 13 \\
\hline MV for $>48$ hours for $<90 \%{ }^{e}$ & 41 & 0 & 9 & 16 & 6 \\
\hline PPAP for control groups & 0 & 0 & 0 & 10 & 0 \\
\hline Trauma ICUs ${ }^{f}$ & 25 & 8 & 3 & 13 & 1 \\
\hline CRF as selection criteria ${ }^{g}$ & 11 & 0 & 0 & 11 & 6 \\
\hline Paediatric ICU & & & 1 & 1 & \\
\hline North American ICU & 36 & 10 & 8 & 6 & 3 \\
\hline $\begin{array}{l}\text { Study publication year } \\
\text { (range) }\end{array}$ & 1987-2019 & $1987-2017$ & $\begin{array}{l}2000- \\
2018\end{array}$ & $\begin{array}{l}1984- \\
2021\end{array}$ & $\begin{array}{l}1994- \\
2014\end{array}$ \\
\hline \multicolumn{6}{|l|}{ Group characteristics } \\
\hline Number of groups ${ }^{d}$ & 166 & 88 & 37 & 131 & 32 \\
\hline \multirow{2}{*}{$\begin{array}{l}\text { Numbers of patients per } \\
\text { study group; median (IQR) } h\end{array}$} & 280 & 75 & 130 & 47 & 69 \\
\hline & $118-596$ & $61-143$ & $72-347$ & $31-72$ & $49-78$ \\
\hline $\begin{array}{l}\text { Mean Length of stay }<7 \text { days; } \\
\text { (number of groups) }\end{array}$ & 27 & 14 & 12 & 14 & 2 \\
\hline $\begin{array}{l}\text { Candidemia risk factors; } \\
\text { (number of groups) }\end{array}$ & 11 & 0 & 0 & 21 & 14 \\
\hline \multicolumn{6}{|l|}{$\begin{array}{l}\text { Indicative intervention effect } \\
\text { size (VAP / RT candida) })^{i, j}\end{array}$} \\
\hline $\begin{array}{l}\text { VAP Pseudomonas } \\
\text { prevention effect (fiqure s5) }\end{array}$ & NA & 0.75 & 0.61 & 0.33 & NR \\
\hline (odds ratio; 95\% Cl; n) & & $\begin{array}{l}0.61-0.91 \\
(39)\end{array}$ & $\begin{array}{l}0.38- \\
0.97 \\
(11)\end{array}$ & $\begin{array}{l}0.26- \\
0.42 \\
(39)\end{array}$ & \\
\hline $\begin{array}{l}\text { RT candida prevention effect } \\
\text { (figure s9) }\end{array}$ & NA & $\begin{array}{l}0.62 \\
0.42-0.9\end{array}$ & 0.37 & 0.54 & NR \\
\hline
\end{tabular}


Table 1. Characteristics of studies (continued)

\begin{tabular}{|c|c|c|c|c|c|}
\hline & Observational & $\begin{array}{l}\text { Non- } \\
\text { decontamination }\end{array}$ & $\begin{array}{l}\text { Topical } \\
\text { anti- } \\
\text { septic }\end{array}$ & $\begin{array}{l}\text { Antibiotic } \\
\text { based }\end{array}$ & $\begin{array}{l}\text { Single } \\
\text { anti- } \\
\text { fungal }\end{array}$ \\
\hline \multicolumn{6}{|l|}{$\begin{array}{l}\text { Indicative intervention effect } \\
\text { size }^{i, k} \\
\text { (Bacteremia/Candidemia) }\end{array}$} \\
\hline \multirow{3}{*}{$\begin{array}{l}\text { Pseudomonas bacteremia } \\
\text { prevention effect (figure s6) } \\
\text { (odds ratio; } 95 \% \mathrm{Cl} ; \mathrm{n} \text { ) }\end{array}$} & \multirow[t]{3}{*}{ NA } & 7.46; & 1.0 & 0.82 & \multirow[t]{3}{*}{ NR } \\
\hline & & \multirow{2}{*}{$\begin{array}{l}0.47-120 \\
(1)\end{array}$} & $\begin{array}{l}0.67- \\
1.5\end{array}$ & $\begin{array}{l}0.52- \\
1.29\end{array}$ & \\
\hline & & & (7) & (19) & \\
\hline \multirow{3}{*}{$\begin{array}{l}\text { Candidemia prevention effect } \\
\text { (figure s10) } \\
\text { (odds ratio; } 95 \% \mathrm{Cl} ; \mathrm{n} \text { ) }\end{array}$} & \multirow[t]{3}{*}{ NA } & 1.01; & 0.75 & 0.48 & 0.43 \\
\hline & & \multirow{2}{*}{$\begin{array}{l}0.06-16.1 \\
(1)\end{array}$} & $0.55-$ & $0.27-$ & $0.23-$ \\
\hline & & & (7) & (17) & (16) \\
\hline
\end{tabular}

1. Among anti-septic studies, topical chlorhexidine was used in 15 of 20 intervention groups.

2. Among TAP intervention groups, the most common antibiotic combination used were polymyxin in combination with an aminoglycoside in 62 of 84 groups. Also, a topical anti-fungal was used in all but eight interventions groups, with amphotericin being the most common anti-fungal ( 50 intervention groups).

3. Fluconazole was the most common single agent antifungal, used in seven intervention groups.

4. Note, several studies had more than one control and or intervention group. Hence the number of groups does not equal the number of studies

5. Studies for which less than $90 \%$ of patients were reported to receive $>48$ hours of MV

6. Trauma ICU arbitrarily defined as an ICU with more than $50 \%$ of admissions for trauma.

7. Use of Candidemia risk factors (CRF) as study inclusion criteria

8. Data is median and inter-quartile range (IQR)

9. Note that studies with zero events in both control and intervention arms do not contribute in the calculation of summary effects size. 
10. Effect size is indicative for each category. Anti-septic interventions include Iseganin in one study; TAP interventions were usually in combinations with an anti-fungal agent; SAF interventions were single include nystatin and TAP in one study and fluconazole in combinations with TAP in another study.

11. Effect size is indicative as several interventions with combinations of agents have been included. TAP interventions were usually in combinations with an anti-fungal agent; SAF interventions include either nystatin (six intervention groups) or fluconazole or another agent (nine intervention groups).

12. Summary effect size from 7 studies that used nystatin was $1.2(0.79-1.83)$ and from 9 studies that used an azole as SAF was $0.21(0.11-0.4)$.

\section{Figures}

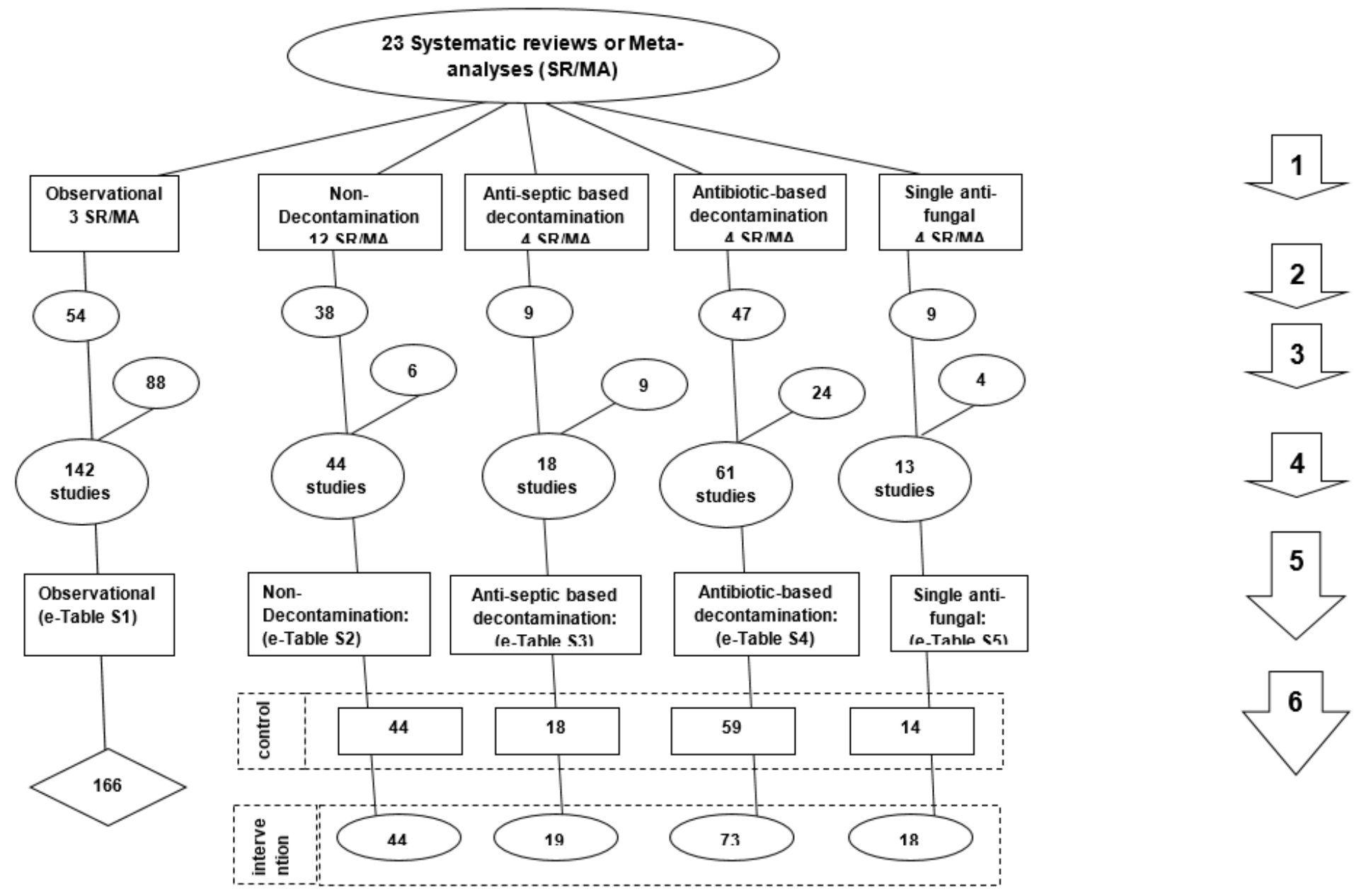

\section{Figure 1}

Search method, screening criteria and resulting classification of eligible studies and subsequent decant of component groups. The four numbered arrows are as follows; (1) An electronic search for systematic reviews or meta-analysis (SR/MA) containing potentially eligible studies using search terms; "ventilator associated pneumonia", "mechanical ventilation", "intensive care unit", each combined with either "meta- 
analysis" or "systematic review" up to December 2018; (2) The systematic reviews were then searched for studies of patient populations requiring prolonged (> 24 hours) ICU admission (3) The studies were triaged from the systematic reviews into one of five categories; studies in which there was no intervention (observational studies), studies of various non-decontamination methods such as methods delivered either via the gastric route, the airway route or via the oral care route, studies of anti-septic methods, studies of antibiotic-based interventions, and studies of single drug antifungal (SAF) prophylaxis. (4) All studies were reviewed for potentially eligible studies and screened against inclusion and exclusion criteria. Any duplicate or ineligible studies were removed and (5) Studies identified outside of systematic reviews were included; (6) The component groups were decanted from each study being control (rectangles), intervention (ovals) and observation (diamond) groups. Note; the total numbers do not tally as some systematic reviews provided studies in more than one category and some studies provided groups in more than one category and some studies have unequal numbers of control and interventions groups. TAP = Topical antibiotic prophylaxis 

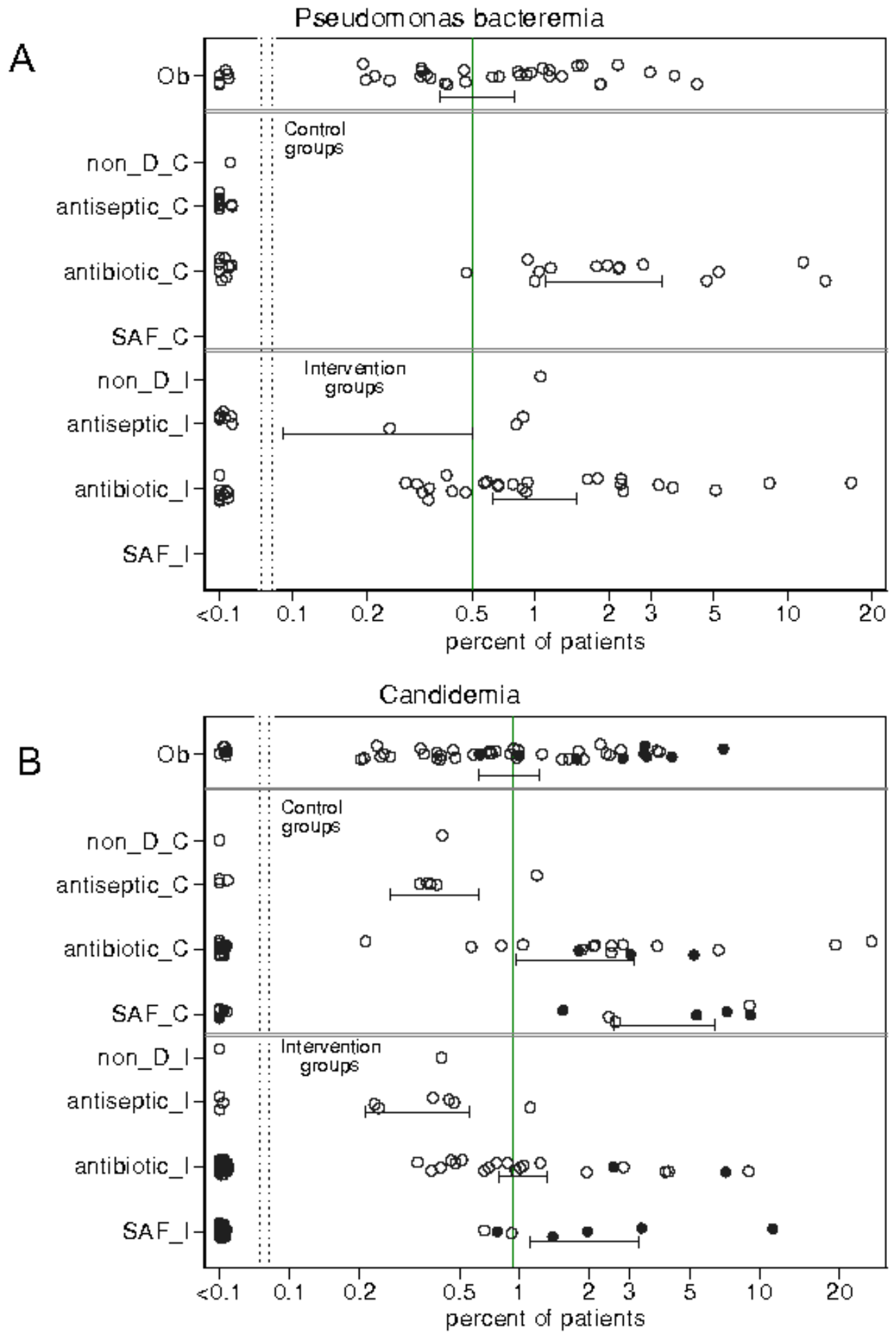

\section{Figure 2}

(a \& b). Scatter plots, on a logit scale, of the incidence proportions of Pseudomonas bacteremia (Fig 2a) and candidemia (Fig 2c) for groups from 289 studies as listed in tables S1 to S5. The mean proportion (and $95 \% \mathrm{Cl}$ ) derived by random effect meta-analysis for each category of component (observational [Ob], control [_C] and intervention [_I]) group derived from observational [Ob], non-decontamination (nonD), antibiotic-based and single anti-fungal (SAF) studies, is displayed. In each plot, the benchmark 
proportion (solid vertical line) is the mean proportion derived from the observational groups. Those component groups that did (solid squares) versus did not (open squares) select patients with CRF's are indicated. NCC is non-concurrent control; CC is concurrent control.

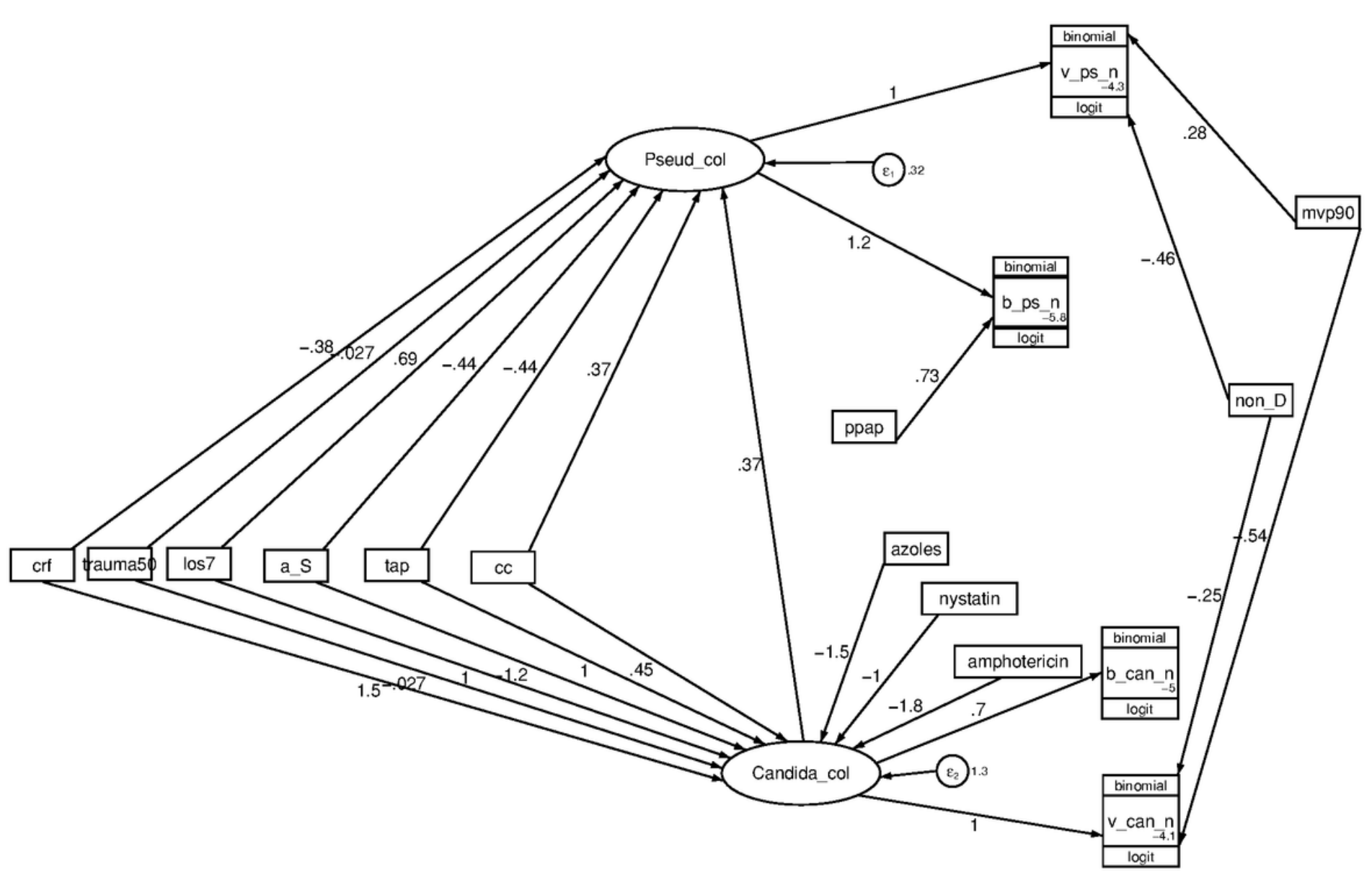

Figure 3

GSEM of the interaction model in relation to Pseudomonas and Candida infection data. Candida col and Pseudomonas col (ovals) are latent variables representing Candida and Pseudomonas colonization, respectively. The variables in rectangles are binary predictor variables representing the group level exposure to the following; trauma ICU setting (trauma50), mean or median length of ICU stay $\geq 7$ days (los7), exposure to a topical anti-septic (a_S), exposure to TAP (tap), concurrency of a control group with an antibiotic-based intervention group (CC), exposure to a non-decontamination based prevention method (non-D), greater than $90 \%$ use of mechanical ventialtion (mvp90) or exposure to PPAP (ppap). Note that the model factorizes exposures from compound regimens (e.g. SDD and SOD, which combine TAP, an antifungal together with or without PPAP) are factorized into singleton TAP, PPAP and anti-fungal exposures. The circles contain error terms. The three part boxes represent the binomial data for Candida and Pseudomonas VAP (v_can_n, v_ps_n) and candidemia (b_can_n) or bacteremia (b_ps_n) counts with 
the number of patients as the denominator which is logit transformed using the logit link function in the generalized model.

A

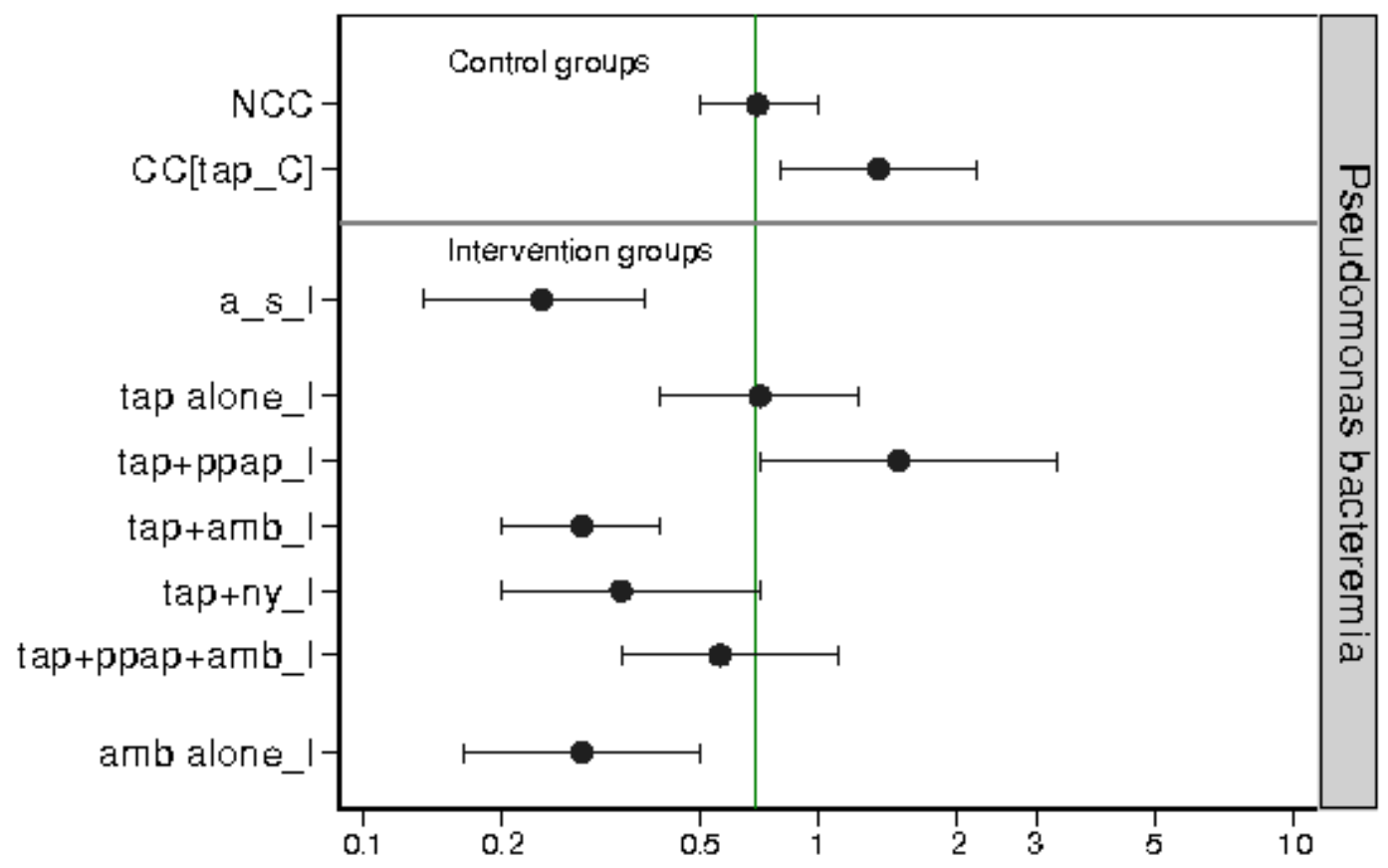

B

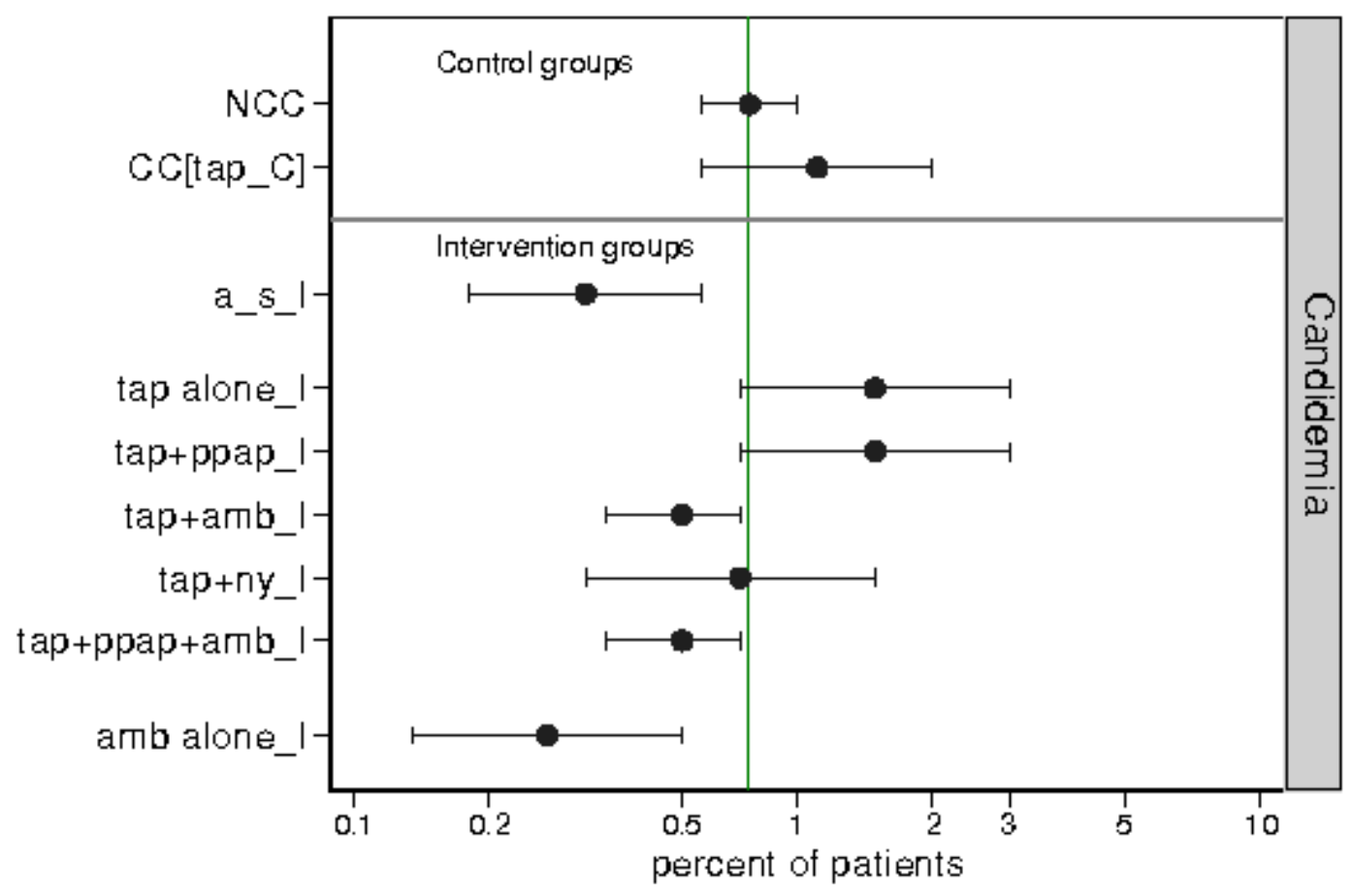

Figure 4

(a \& b). Model predictions derived from model A (fig 3) for the incidence proportions of Pseudomonas bacteremia (Fig 4a), and candidemia (Fig 4b) for a group of patients in a non-trauma ICU with mean LOS $>7$ days without selection for CRF. The projections are for control (top) or intervention (bottom panel) 
groups receiving prophylaxis with various singleton or combination interventions. In each plot, the benchmark proportion (solid vertical line) is the mean prediction derived for an equivalent NCC group without exposures. NCC is non-concurrent control; CC is concurrent control; non-D is non-

decontamination, A_s is anti-septic, TAP is topical antibiotic prophylaxis, amb is amphotericin and ny is nystatin, ppap is protocolized parenteral antibiotic prophylaxis

\section{Supplementary Files}

This is a list of supplementary files associated with this preprint. Click to download.

- STROBEchecklistv4combined.docx

- onlinesuppldysbiosis4.pdf 